\title{
Le ribosome change de piste au cours du cycle cellulaire
}

La progression à travers les différentes phases du cycle cellulaire nécessite l'expression de protéines spécifiques à chacune des phases. Jusqu'à présent, l'analyse des mécanismes de contrôle de l'expression temporelle de ces protéines a révélé le rôle primordial de la transcription et des modifications post-traductionnelles. Pourtant, on sait depuis trente ans que le taux global de traduction n'est pas constant au cours du cycle cellulaire [1, 2]. Il augmente pendant la phase de croissance cellulaire, lors de la transition G1/S, reste stable en $\mathrm{S}$, puis diminue lors de la transition $\mathrm{G} 2 / \mathrm{M}$, et reste à un taux très bas (20\% du taux en G1/S) pendant la mitose. Paradoxalement, la traduction de certains ARNm est sélectivement activée en G2/M, suggérant l'existence d'un mécanisme spécifique. Il n'est donc pas surprenant que deux études publiées récemment dans Molecular Cell [3, 4] aient identifié deux ARNm traduits en mitose par un mécanisme distinct $\mathrm{du}$ mode de biosynthèse classique. On peut s'étonner que trente ans se soient écoulés entre la découverte de l'inhibition générale de la traduction en G2/M et la démonstration d'un mécanisme alternatif permettant d'éviter cette inhibition.

Les premières études disséquant les mécanismes responsables de l'inhibition du taux de traduction en G2/M avaient souligné l'importance de l'étape d'enclenchement de la synthèse protéique, mais la connaissance de la machinerie traductionnelle était alors trop succincte pour fournir une explication moléculaire précise. Ce n'est qu'après la caractérisation des facteurs d'enclenchement tor), que les fluctuations cycliques du taux de traduction ont pu être analysées plus en détail. Chez les eucaryotes, le démarrage de la synthèse des protéines est un processus complexe car il n'existe pas de complémentarité directe entre le ribosome et l'ARNm. Le ribosome est recruté grâce au complexe protéique eIF4F (qui est composé de trois facteurs), lequel relie physiquement l'extrémité coiffée de l'ARNm à la sousunité 40S du ribosome [5]. Un intérêt majeur de ce complexe eIF4F est son caractère contrôlable : toute altération de son intégrité doit modifier l'efficacité de la traduction des ARNm coiffés. Ainsi, les fluctuations cycliques du taux global de traduction ont pu être attribuées, au moins en partie, à des modifications de l'assemblage du complexe eIF4F. Cependant, eIF4F, dont la fonction se résume à attacher la sous-unité $40 \mathrm{~S}$ du ribosome à n'importe quel ARNm coiffé, ne peut exercer de régulation spécifique. Dans ce contexte, on voit mal comment la machinerie traductionnelle pourrait cibler spécifiquement une sous-population d'ARNm dans les cellules en phase en G2/M. Une fois fixé à l'extrémité 5' de l'ARNm, le ribosome doit identifier le codon initiateur. Pour cela, il scrute la région 5' non traduite (modèle du balayage ou scanning) entre la coiffe et le codon initiateur. Cette opération est en général facile car la majorité des ARNm eucaryotes possèdent une région 5' non traduite simple. Cependant, pour la minorité restante, cette région est longue, structurée et parsemée de codons initiateurs que le ribosome doit occulter puisqu'ils sont placés dans un contexte nucléotidique défavorable.
Le balayage du ribosome y est peu efficace et coûteux en énergie. Cette piste chaotique permet cependant aux ARNm d'échapper au contrôle général exercé par le complexe eIF4F : elle est en effet suffisamment complexe pour bloquer la progression du ribosome, et renferme de plus toute l'information nécessaire à un mode spécifique de traduction. Le premier des deux ARNm pour lesquels a été identifié un mode spécifique de traduction en G2/M [3] code pour l'ornithine décarboxylase (ou ODC), l'enzyme-clé de la biosynthèse des polyamines. Les polyamines sont requises lors la réplication de l'ADN en phase $S$, puis en mitose où elles interviennent dans la polymérisation des microtubules et la condensation des chromosomes. Jusqu'à présent, on considérait que la traduction de l'ARNm ODC était très dépendante de la coiffe et qu'elle était réprimée de façon constitutive du fait de la complexité de sa région 5'. L'augmentation de l'expression de l'ODC observée en G1/S est corrélée à l'augmentation globale de la traduction provoquée par l'activation du complexe eIF4F. Un second pic d'expression observé en G2/M restait quant à lui inexpliqué, d'autant plus qu'il survenait pendant l'inhibition générale de la traduction. Notre équipe vient de montrer que la région $5^{\prime}$ non traduite du messager de l'ODC renferme un site d'entrée interne pour le ribosome appelé IRES (pour internal ribosome entry site) [3]. Ce site est indépendant de la coiffe et du complexe eIF4F. La traduction de l'ARNm ODC peut ainsi échapper au contrôle exercé par les facteurs généraux. En effet, l'IRES de l'ODC fonctionne sélectivement lors 
de la transition G2/M, lorsque le complexe eIF4F est inactivé. On peut assimiler cet IRES à une piste alternative, accessible par le ribosome uniquement en G2/M, qui facilite la traduction d'un nombre restreint d'ARNm en court-circuitant la région inhibitrice située en amont (figure 1A).

Le second ARNm contenant un IRES fonctionnel en G2/M décrit dans le même numéro de la revue code pour la protéine mitotique p58 ${ }^{\text {PITSLRE }}$ [4], une kinase dépendante des cyclines (ou cdk). Comme pour l'ODC, l'ARNm de p58 $8^{\text {PITSLRE }}$ est présent tout $\mathrm{au}$ long du cycle cellulaire. Cependant, les mécanismes de déclenchement alternativement utilisés au cours du cycle y ont des conséquences différentes. Une autre protéine, p110 $10^{\text {PITSLRE }}$, est en effet synthétisée à partir du même transcrit tout au long du cycle cellulaire, mais sa synthèse débute au niveau du premier codon initiateur AUG de la région 5' non traduite selon le mécanisme classique dépendant de la coiffe. En revanche, la synthèse de la protéine p5 $58^{\text {PITSLRE }}$ débute au niveau $\mathrm{du} 18^{\mathrm{e}}$ AUG, et uniquement en G2/M. Dans ce cas, l'utilisation de l'IRES permet non seulement d'échapper au contrôle exercé par le complexe eIF4F, mais aussi d'occulter les 17 premiers AUG afin de synthétiser une protéine tronquée de son extrémité amino-terminale (figure 1B).

Ainsi, l'augmentation du taux global de traduction dépendante de la coiffe reflète l'activation du complexe eIF4F pendant la phase G1/S de croissance du cycle cellulaire. Le mécanisme d' "entrée interne du ribosome» est, quant à lui, mis en

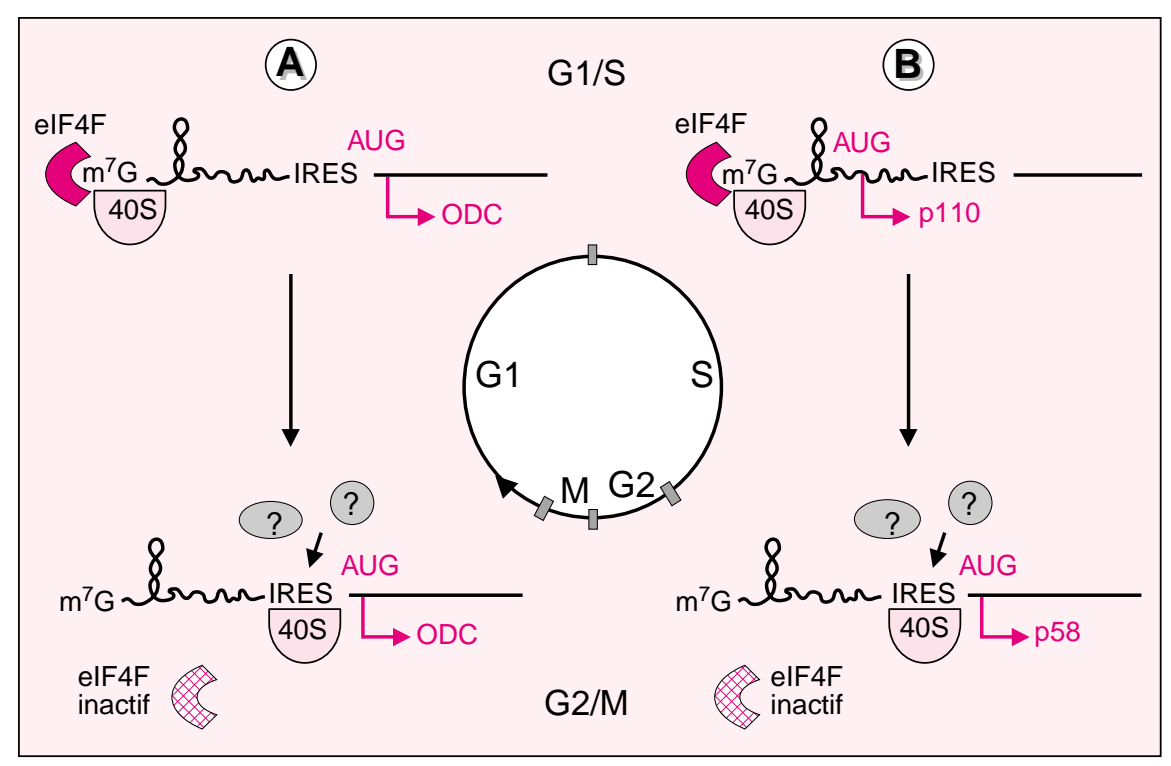

Figure 1. Les deux modes de traduction au cours du cycle cellulaire. Le cercle représente un cycle cellulaire complet. En G1/S, la traduction est dépendante de la coiffe ( $\mathrm{m}^{7} \mathrm{Gppp}$ ). Grâce au complexe elF4F, la coiffe recrute la sous-unité $40 S$ du ribosome au niveau de l'extrémité $5^{\prime}$ de l'ARNm. La progression de la sous-unité $40 S$ est alors sensible à la nature de la séquence non traduite comprise entre la coiffe et le codon AUG placé dans un contexte favorable au déclenchement de la traduction. En G2/M, le complexe elF4F est inactivé et le démarrage de la traduction est lié à l'entrée interne du ribosome (IRES). Ce mécanisme permet de court-circuiter les séquences situées en amont dans la région $5^{\prime}$ non traduite. II permet la synthèse soit de la même protéine $(O D C, A)$, soit d'une protéine tronquée de son extrémité $N$ terminale (p58, B). II est vraisemblable que des facteurs encore inconnus interviennent dans ce processus. œuvre sélectivement en G2/M et permet la synthèse des protéines requises en mitose. La connaissance des modes d'activation de ces IRES au cours du cycle sera déterminante pour concevoir des molécules (agents antitumoraux par exemple) permettant de bloquer la synthèse d'un groupe défini de protéines nécessaires à la progression vers la mitose. Une étude récente, fondée sur la quantification par la technique de "microarray» des ARNm enclenchés dans les polysomes malgré l'inactivation du complexe eIF4F permet d'estimer à environ 200 (sur les 7000 testés) les ARNm susceptibles d'emprunter ce mécanisme alternatif [6].

Les premiers IRES ont été découverts, il y a plus de dix ans, chez les picornavirus [7]. Ces virus ne codant pas pour les protéines responsables du démarrage de la traduction, on a alors pensé que les ARN viraux usurpaient un mécanisme déjà existant dans la cellule hôte. Ultérieurement, l'identification d'IRES dans divers ARNm cellulaires a renforcé cette hypothèse. Cependant, plus de dix ans après sa mise en évidence, le principe moléculaire responsable de l'entrée interne demeure un mystère. Les IRES peuvent aussi être fonctionnels dans certaines situations de stress, comme les infections virales [7], le stress oxydatif [8] ou les rayonnements [9] et lors du processus apoptotique [10]. Dans ces situations, comme pendant la mitose, l'intégrité du complexe eIF4F est altérée, et il est donc probable que l'entrée interne profite de cette inactivation du complexe eIF4F. Autrement dit, il pourrait exister une compétition entre la traduction dépendant de la coiffe et celle utilisant un IRES pour l'utilisation de facteurs communs. Néanmoins, l'intervention de protéines régulatrices (en trans) capables de reconnaître des séquences spécifiques portées par les ARNm (en cis) n'est pas exclue [11]. De nombreuses équipes recherchent les mécanismes et facteurs inducteurs de l'utilisation de cette deuxième piste. 
1. Fan H, Penman S. Regulation of protein synthesis in mammalian cells. J Mol Biol 1970; 50: 655-70.

2. Tarnowka MA, Baglioni C. Regulation of protein synthesis in mitotic HeLa cells. J Cell Physiol 1979; 99 : 359-68.

3. Pyronnet S, Pradayrol L, Sonenberg N. A cell cycle-dependent internal ribosome entry site. Mol Cell 2000; 5 : 607-16.

4. Cornelis S, Bruynooghe Y, Denecker G, Van Huffel S, Tinton S, Beyaert R. Identification and characterization of a novel cell cycle-regulated internal ribosome entry site. Mol Cell 2000; 5 : 597-605.

5. Poulin F, Pyronnet S. Interactions moléculaires et initiation de la synthèse protéique. Med Sci $2000 ; 16: 617-22$.

6. Johannes G, Carter M, Eisen M, Brown P, Sarnow P. Identification of eukaryotic mRNAs that are translated at reduced cap binding complex eIF4F concentrations using a cDNA microarray. Proc Natl Acad Sci USA 2000; 96 : 13118-23.

7. Pelletier J, Sonenberg N. Internal initiation of translation of eukaryotic mRNA directed by a sequence derived from poliovirus RNA. Nature $1988 ; 334: 320-5$.

8. Vagner S, Touriol C, Galy B, et al. Translation of CUG- but not AUG-initiated forms of human fibroblast growth factor 2 is activated in transformed and stressed cells. J Cell Biol 1996; 135 : 1391-402.

9. Holcik M, Lefebvre C, Yeh C, Chow T, Korneluk RG. A novel internal ribosome entry site (IRES) motif potentiates XIAP mediated cytoprotection. Nat Cell Biol 1999; 3: 190-3.

10. Henis-Korenblit S, Strumpf NL, Golgstaub D, Kimchi A. A novel form of DAP5 protein accumulates in apoptotic cells as a result of caspase cleavage and internal ribosome entry site-mediated translation. Mol Cell Biol 2000; 20 : 496-506. 11. Sachs AB. Cell cycle-dependent translation initiation: IRES Elements Prevail. Cell 2000; 101 . 243-5.

\section{Stéphane Pyronnet Lucien Pradayrol}

Biologie et pathologie digestive, Inserm U. 531, Institut Louis-Bugnard, CHU Rangueil, 31403 Toulouse Cedex, France. 